\title{
Real-Time Model-Based Fault Diagnosis for Switching Power Converters
}

\author{
Jason Poon, Ioannis C. Konstantakopoulos, Costas Spanos, Seth R. Sanders \\ Department of EECS \\ University of California, Berkeley \\ Berkeley, CA 94720 \\ Email: jason@berkeley.edu
}

\begin{abstract}
We present the analysis, design, and experimental implementation of a fault diagnosis method for switching power converters using a model-based estimator approach. The fault diagnosis method enables efficient detection and identification of component and sensor faults, and is implemented on the same computation platform as the control system. The modelbased estimator operates in parallel with the switching power converter, and generates an error residual vector that can be used to detect and identify particular component or sensor faults. This paper presents an experimental demonstration for a $1.2 \mathbf{k W}$ rack-level uninterruptable power supply (UPS) dc-dc converter for data center applications. Simulation and experimental results demonstrate fault detection and identification for various component and sensor faults in the converter. Moreover, we show that the proposed fault diagnosis design and analysis methods are applicable to a broad class of converter topologies and fault types.
\end{abstract}

\section{INTRODUCTION}

In recent years, there have been renewed efforts towards realizing more efficient and less redundant data center power distribution systems, while still maintaining high reliability and fault tolerance. In many safety-critical and high-availability application domains, model-based fault diagnosis is an effective method of achieving these goals while minimizing additional costs and system complexity [1]-[3].

In existing works, model-based fault diagnosis has been successfully applied in certain switching power converter applications [4]-[8]. However, most of these approaches target specific faults that occur in a particular converter topology, such as a short-circuit switch fault in a three-phase voltagesource inverter, and are not easily expandable to different converter topologies or fault types.

Recent works have made progress towards a generalized framework for model-based fault diagnosis in switching power converters. In [9], [10], the authors propose a switched linear observer-based approach for fault detection and identification in three-phase ac-dc power electronics systems using an auxiliary computation platform to solve the observer in real-time.

In this paper, we extend this framework by considering a model-based estimator with no output injection. Moreover, the estimator is implemented on the same computation platform as the control system, thus requiring no additional hardware. The proposed fault diagnosis method complements existing fault protection schemes, in essence, adding a layer of 'intelligence' on top of existing protection hardware and controls.
The fault diagnosis method enables efficient detection and identification of arbitrary component and sensor faults, and can be generalized for a broad class of converter topologies and fault types. We present a real-time digital implementation of the proposed fault diagnosis method for a $1.2 \mathrm{~kW}$ racklevel uninterruptable power supply (UPS) dc-dc converter for data center applications. Simulation and experimental results demonstrate successful fault detection and identification for a set of component and sensor faults in the dc-dc converter.

The remainder of the paper is organized as follows. First, Section II presents the design of the fault diagnosis method for a rack-level UPS module converter. We analyze the faulted converter dynamics to construct a set of 'fault signatures,' which can be used to detect and identify converter faults. Section III presents an experimental implementation of the fault diagnosis method using a field-programmable gate array (FPGA) device that performs the converter control and solves the model-based estimator in real-time. Section IV presents a generalization of the fault diagnosis method to a broad class of switching power converters. Section V concludes the paper.

\section{Design Methodology FOR RaCK-LeVEl UPS DC-DC CONVERTER}

In this section, we present the design methodology for model-based fault diagnosis for the rack-level UPS dc-dc converter shown in Figure 1. The complete specifications for this converter are presented in Table I.

The basic principles of operation of the fault diagnosis method are as follows. First, given a switching power converter, we construct a linear time-varying dynamical model that captures the large-signal dynamics of the converter. Next, we identify faults of interest in the components and sensors. The dynamics of each of these faults can be uniquely modeled by a scalar fault magnitude function and a vector fault signature. We construct a model-based estimator for the switching power converter, which generates an error residual vector of the difference between the measured outputs of the converter and the estimated outputs. We can show that the open-loop error dynamics are stable (see [11]). Moreover, due to the lossiness in the converter and corresponding model, the error residual is zero in the steady state for a fault-free system. In the presence of a particular fault, the error residual will evolve according to the dynamics of the fault magnitude function 


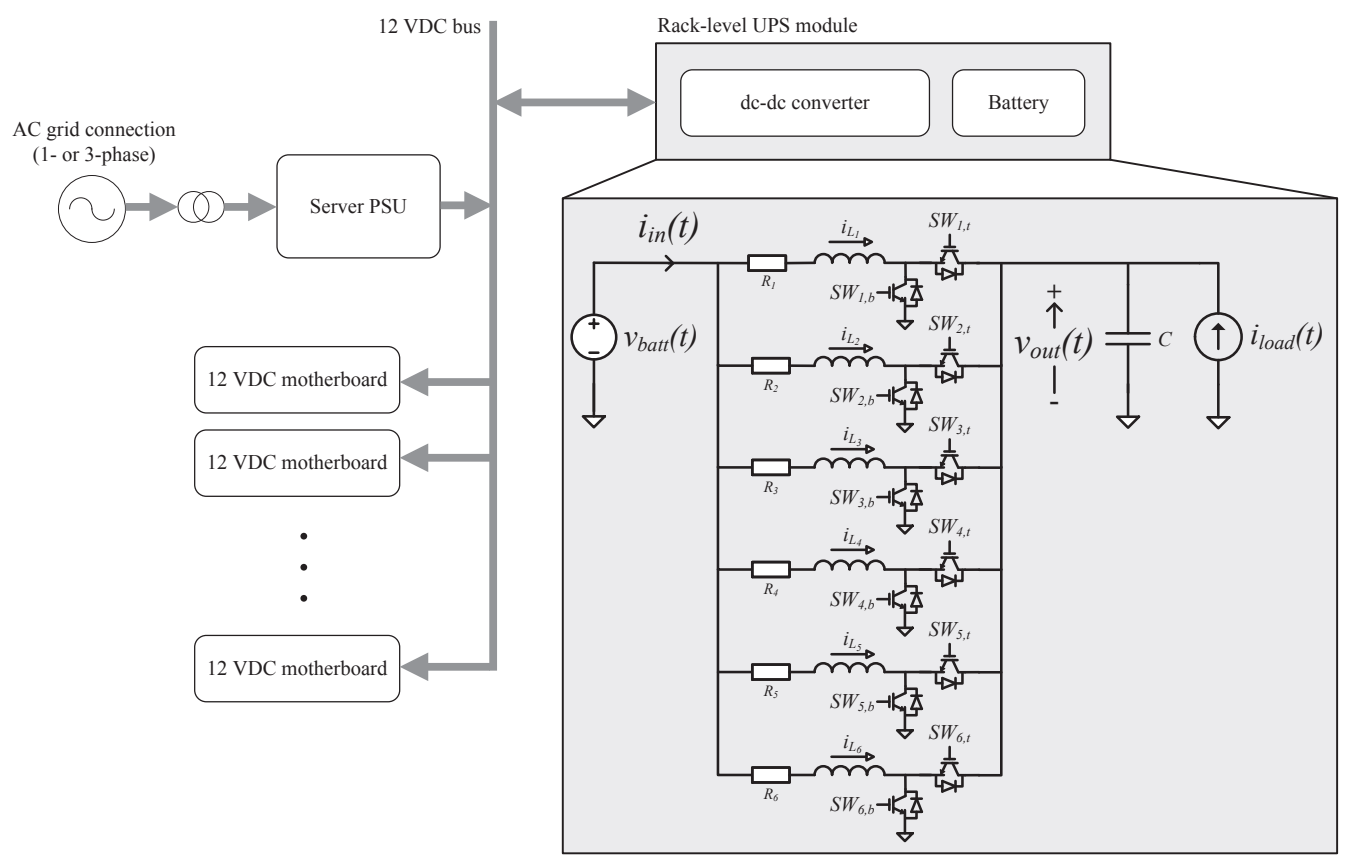

Fig. 1: A data center power distribution network configuration. The converter topology of the rack-level UPS module is shown.

TABLE I: Specifications for conveter experimental testbed.

$\begin{array}{ll}\text { Switching power converter } & \\ \text { Topology } & \text { 6-phase boost converter } \\ \text { Rated output voltage } & 11.8 \mathrm{~V} \\ \text { Rated output current } & 100 \mathrm{~A} \\ R_{n} & 0.1 \Omega \\ L_{n} & 0.1 \mathrm{mH} \\ C & 400 \mu \mathrm{F} \\ \text { Battery pack } & \mathrm{K} 2 \mathrm{Energy} \mathrm{LFP26650EV} \\ \text { Battery cell } & 9.6 \mathrm{~V} \\ \text { Nominal } v_{\text {batt }} & 25.6 \mathrm{~A}-\mathrm{h} \\ \text { Pack capacity } & \\ \text { Real-time computation platform } & \text { Xilinx Virtex-6 ML605 } \\ \text { FPGA device } & 500 \mathrm{~ns} \\ \text { Estimator solver time step } & 1 \mathrm{MHz} \\ \text { Analog input sampling rate } & 10 \mathrm{kHz} \\ \text { PWM switching frequency } & 50\end{array}$

and fault signature. Since these dynamics are calculated a priori, we can efficiently detect and identify the fault by computing the sliding window $L^{2}$-inner product between the error residual vector and the set of fault signatures.

\section{A. Converter modeling}

First, we construct a linear-time varying model of the dcdc converter operating in continuous conduction mode. We assume that the switches $S W_{n, t}$ and $S W_{n, b}$ for $n=1 \ldots 6$ are ideal, and are controlled by an ideal complementary switching signal vector $k(t)$, where $k_{n}(t)=1$ indicates that $S W_{n, t}$ is 'on' and $S W_{n, b}$ is 'off', while $k_{n}(t)=0$ indicates that $S W_{n, t}$ is 'off' and $S W_{n, b}$ is 'on'. Thus, the dynamical model of the converter is:

$$
\begin{aligned}
\frac{d x(t)}{d t} & =A(t) x(t)+B u(t), \\
y(t) & =H x(t),
\end{aligned}
$$

where

$$
\begin{gathered}
x(t)=\left[\begin{array}{c}
i_{L_{1}} \\
i_{L_{2}} \\
i_{L_{3}} \\
i_{L_{4}} \\
i_{L_{5}} \\
i_{L_{6}} \\
v_{C}
\end{array}\right], u(t)=\left[\begin{array}{c}
v_{\text {batt }}(t) \\
i_{\text {load }}(t)
\end{array}\right], y(t)=\left[\begin{array}{c}
i_{\text {in }}(t) \\
v_{\text {out }}(t)
\end{array}\right] \\
A(t)=\left[\begin{array}{cccccccc}
-\frac{R}{L} & 0 & 0 & 0 & 0 & 0 & -\frac{k_{1}(t)}{L} \\
0 & -\frac{R}{L} & 0 & 0 & 0 & 0 & -\frac{k_{2}(t)}{L} \\
0 & 0 & -\frac{R}{L} & 0 & 0 & 0 & -\frac{k_{3}(t)}{L} \\
0 & 0 & 0 & -\frac{R}{L} & 0 & 0 & -\frac{k_{4}(t)}{L} \\
0 & 0 & 0 & 0 & -\frac{R}{L} & 0 & -\frac{k_{5}(t)}{L} \\
0 & 0 & 0 & 0 & 0 & -\frac{R}{L} & -\frac{k_{6}(t)}{L} \\
\frac{k_{1}(t)}{C} & \frac{k_{2}(t)}{C} & \frac{k_{3}(t)}{C} & \frac{k_{4}(t)}{C} & \frac{k_{5}(t)}{C} & \frac{k_{6}(t)}{C} & 0
\end{array}\right], \\
B=\left[\begin{array}{cc}
\frac{1}{L} & 0 \\
\frac{1}{L} & 0 \\
\frac{1}{L} & 0 \\
\frac{1}{L} & 0 \\
\frac{1}{L} & 0 \\
\frac{1}{L} & 0 \\
0 & \frac{1}{C}
\end{array}\right], H=\left[\begin{array}{lllllll}
1 & 1 & 1 & 1 & 1 & 1 & 0 \\
0 & 0 & 0 & 0 & 0 & 0 & 1
\end{array}\right]
\end{gathered}
$$

\section{B. Fault modeling}

Next, we model the dynamics of the system under faulted conditions. Consider a fault in the inductor $L_{n}$ of the $n^{t h}$ phase of the converter that causes the value of the inductance to change by a quantity $\Delta L$. The fault manifests as an additive 
TABLE II: Component fault in $L_{n}$ causing $\Delta L$ change in inductance.

\begin{tabular}{ccc}
\hline Component & $\phi_{i}$ & $f_{i}$ \\
\hline$L_{1}$ & $\frac{R \Delta L i_{L_{1}}(t)+k_{1}(t) \Delta L v_{C}(t)-\Delta L v_{\text {batt }}(t)}{L(L+\Delta L)}$ & {$[1,0,0,0,0,0,0]^{T}$} \\
$L_{2}$ & $\frac{R \Delta L i_{L_{2}}(t)+k_{2}(t) \Delta L v_{C}(t)-\Delta L v_{\text {batt }}(t)}{L(L+\Delta L)}$ & {$[0,1,0,0,0,0,0]^{T}$} \\
$L_{3}$ & $\frac{R \Delta L i_{L_{3}}(t)+k_{3}(t) \Delta L v_{C}(t)-\Delta L v_{\text {batt }}(t)}{L(L+\Delta L)}$ & {$[0,0,1,0,0,0,0]^{T}$} \\
$L_{4}$ & $\frac{R \Delta L i_{L_{4}}(t)+k_{4}(t) \Delta L v_{C}(t)-\Delta L v_{\text {batt }}(t)}{L(L+\Delta L)}$ & {$[0,0,0,1,0,0,0]^{T}$} \\
$L_{5}$ & $\frac{R \Delta L i_{L_{5}}(t)+k_{5}(t) \Delta L v_{C}(t)-\Delta L v_{\text {batt }}(t)}{L(L+\Delta L)}$ & {$[0,0,0,0,1,0,0]^{T}$} \\
$L_{6}$ & $\frac{R \Delta L i_{L_{6}}(t)+k_{6}(t) \Delta L v_{C}(t)-\Delta L v_{\text {batt }}(t)}{L(L+\Delta L)}$ & {$[0,0,0,0,0,1,0]^{T}$} \\
\hline
\end{tabular}

TABLE III: Component fault in $C$ causing $\Delta C$ change in capacitance.

\begin{tabular}{ccc}
\hline Component & $\phi_{i}$ & $f_{i}$ \\
\hline$C$ & $-\frac{\Delta C}{C(C+\Delta C)} i_{l o a d}(t)-\sum_{n=1}^{6} \frac{\Delta C k_{n}(t)}{C(C+\Delta C)} i_{L_{n}}(t)$ & {$[0,0,0,0,0,0,1]^{T}$} \\
\hline
\end{tabular}

term $\Delta A$ and $\Delta B$ in $A(t)$ and $B$, respectively. Thus, the dynamics of the converter in the presence of this fault are:

$$
\frac{d x(t)}{d t}=(A(t)+\Delta A) x(t)+(B+\Delta B) u(t)
$$

With simple algebraic manipulation, we can rewrite (3) as the sum of (1) and the product of a scalar component fault magnitude function $\phi_{i}$ and a vector component fault signature $f_{i}$, that is:

$$
\frac{d x(t)}{d t}=A(t) x(t)+B u(t)+\phi_{i} f_{i},
$$

where, in the case $n=1$,

$$
\begin{aligned}
\phi_{i} & =\frac{R \Delta L i_{L_{1}}(t)+k_{1}(t) \Delta L v_{C}(t)-\Delta L v_{\text {batt }}(t)}{L(L+\Delta L)}, \\
f_{i} & =[1,0,0,0,0,0,0]^{T}
\end{aligned}
$$

We can calculate $\phi_{i}$ and $f_{i}$ for $n=1 \ldots 6$, as shown in Table II. Similarly, we can use the same process to determine $\phi_{i}$ and $f_{i}$ for faults that affect the capacitance of the output capacitor $C$ and for faults in a switch pair that force $S W_{n, t} \rightarrow$ 'off' and $S W_{n, b} \rightarrow$ 'on'. The results of these derivations are shown in Tables III and IV.

Sensor faults manifest differently in the system dynamics than component faults. For example, consider the effect of a fault in the input current $\left(i_{i n}\right)$ sensor that causes a perturbation in the sensor gain $\Delta G_{1}$ and in the sensor offset $\Delta E_{1}$. The fault manifests as an additive term $\Delta H$ and $\Delta E_{j}$ in the output readout map as follows:

$$
y(t)=(H+\Delta H) x(t)+\Delta E_{j}
$$

We can rewrite (5) as the sum of (2) and the product of a scalar sensor fault magnitude function $\theta_{j}$ and a vector sensor
TABLE IV: Fault in switch pair forcing $S W_{n, t} \rightarrow$ 'off' and $S W_{n, b} \rightarrow$ 'on'.

\begin{tabular}{ccc}
\hline Component & \multicolumn{1}{c}{$\phi_{i}$} & $f_{i}$ \\
\hline$S W_{1, t / b}$ & $k_{1}(t)$ & {$\left[\frac{1}{L} v_{C}, 0,0,0,0,0,-\frac{1}{C} i_{L_{1}}\right]^{T}$} \\
$S W_{2, t / b}$ & $k_{2}(t)$ & {$\left[0, \frac{1}{L} v_{C}, 0,0,0,0,-\frac{1}{C} i_{L_{2}}\right]^{T}$} \\
$S W_{3, t / b}$ & $k_{3}(t)$ & {$\left[0,0, \frac{1}{L} v_{C}, 0,0,0,-\frac{1}{C} i_{L_{3}}\right]^{T}$} \\
$S W_{4, t / b}$ & $k_{4}(t)$ & {$\left[0,0,0, \frac{1}{L} v_{C}, 0,0,-\frac{1}{C} i_{L_{4}}\right]^{T}$} \\
$S W_{5, t / b}$ & $k_{5}(t)$ & {$\left[0,0,0,0, \frac{1}{L} v_{C}, 0,-\frac{1}{C} i_{L_{5}}\right]^{T}$} \\
$S W_{6, t / b}$ & $k_{6}(t)$ & {$\left[0,0,0,0,0, \frac{1}{L} v_{C},-\frac{1}{C} i_{L_{6}}\right]^{T}$} \\
\hline
\end{tabular}

TABLE V: Fault in sensor affecting sensor gain and offset.

\begin{tabular}{ccc}
\hline Sensor & $\theta_{j}$ & $g_{j}$ \\
\hline$i_{\text {in }}$ & $\Delta G_{1}(t) i_{\text {in }}(t)+\Delta E_{1}(t)$ & {$[1,0]^{T}$} \\
$v_{\text {out }}$ & $\Delta G_{2}(t) v_{\text {out }}(t)+\Delta E_{2}(t)$ & {$[0,1]^{T}$} \\
\hline
\end{tabular}

fault signature $g_{j}$, that is:

$$
y(t)=H x(t)+\theta_{j} g_{j}
$$

where

$$
\begin{aligned}
\theta_{j} & =\Delta G_{1}(t) i_{i n}(t)+\Delta E_{1}(t), \\
g_{j} & =[1,0]^{T}
\end{aligned}
$$

We can use the same process to determine $\theta_{j}$ and $g_{j}$ for faults in the output voltage sensor $\left(v_{\text {out }}\right)$ as shown in Table $\mathrm{V}$.

\section{Estimator design and implementation}

From Section II-B, we see that the state space dynamics of the switching power converter contain valuable information, particularly the fault magnitude function and fault signature, that can be used to detect and identify faults. Thus, we 


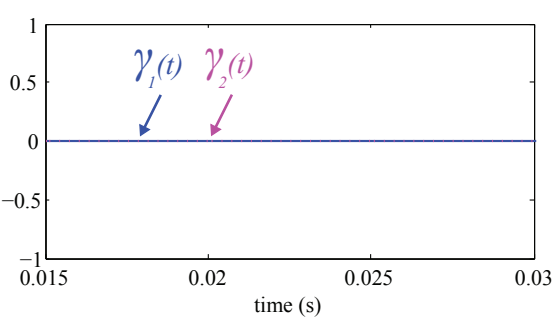

(a) Nominal (fault-free) response.

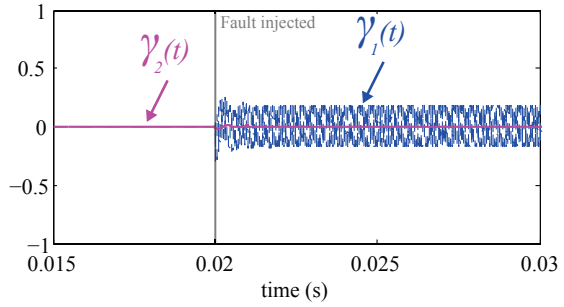

(b) Component fault causing 50 percent reduction of inductance $L_{6}$.

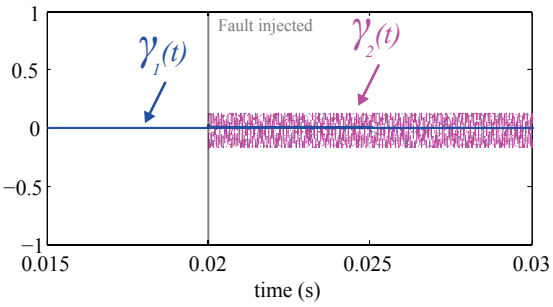

(c) Component fault causing 50 percent reduction of capacitance $C$.

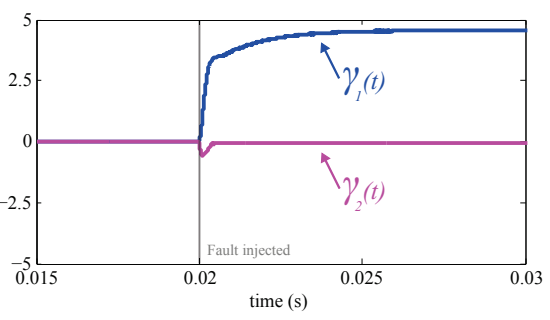

(d) Fault in switch pair forcing $S W_{6, t}$ to 'off' and $S W_{6, b}$ to 'on'.

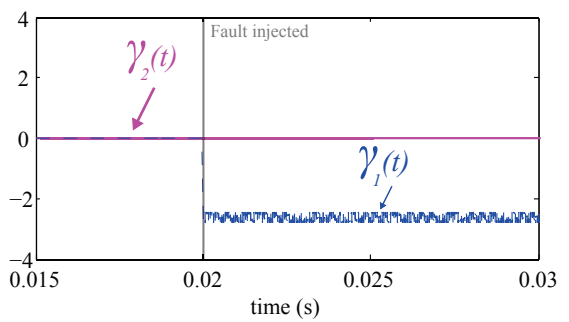

(e) Fault forcing sensor gain of $i_{i n}$ to zero.

Fig. 2: Simulation of estimator response in nominal and component and sensor fault states.

TABLE VI: Normalized fault signatures for component and sensor faults (note that $\left.\xi=\left(\frac{v_{C}(t)^{2}}{L^{2}}+\frac{i_{L_{n}}(t)^{2}}{C^{2}}\right)^{-\frac{1}{2}}\right)$.

\begin{tabular}{cc}
\hline Faulted component & $\overline{H f_{i}}$ \\
\hline$L_{n}$ & {$[1,0]^{T}$} \\
$C$ & {$[0,1]^{T}$} \\
$S W_{n, t / b}$ & $\xi\left[\frac{v_{C}(t)}{L},-\frac{i_{L_{n}(t)}}{C}\right]^{T}$ \\
\hline Faulted sensor & $\overline{g_{j}}$ \\
\hline$i_{\text {in }}$ & {$[1,0]^{T}$} \\
$v_{\text {out }}$ & {$[0,1]^{T}$} \\
\hline
\end{tabular}

propose a model-based estimator that can be used to isolate these components from the measured outputs of the converter. Consider a switched linear estimator of the following form:

$$
\begin{aligned}
\frac{d \hat{x}(t)}{d t} & =A(t) \hat{x}(t)+B u(t) \\
\gamma(t) & =y(t)-H \hat{x}(t)
\end{aligned}
$$

where $\hat{x}(t)$ is an estimate of the state vector $x(t), \gamma(t)$ is the error residual vector, and $A(t), B$, and $H$ are the state space matrices that describe the ideal converter dynamics. In some applications, one might use output injection to compensate for parameter uncertainty and non-linearities that occur in $A(t)$, $B$, and $H$. However, we deliberately eschew this approach, and will demonstrate that these discrepancies naturally manifest as components of the fault magnitude function and fault signature.

The dc-dc converter and model-based estimator are simu- lated in MATLAB/Simulink using the Piecewise Linear Electrical Circuit Simulation (PLECS) toolbox [12].

Fig. 2a shows the nominal (fault-free) response of the error residual vector. Due to the modeled lossiness in the estimator and the natural lossiness in the converter, we have that the error residual vector is zero in steady state.

Next, we introduce faults in the converter and analyze the dynamics of the error residual vector. Using the analysis of the faulted response of the converter presented in Section II-B, we can construct a normalized fault signature, which is the normalized vector function of $H f_{i}$ and $g_{j}$. The normalized fault signature, denoted as $\overline{H f_{i}}$ for component faults and $\overline{g_{j}}$ for sensor faults, is an element of the output readout vector function space, as shown in Table VI. We take the $L^{2}$-inner product between the error residual vector and the set of all normalized fault signatures on an interval $[t-W, t]$, where $W$ is the duration of the interval. We select $W$ to be roughly ten switching cycles of the converter, which enables the inner product calculation to reach steady state in around $1 \mathrm{~ms}$. For components faults, the inner product is:

$$
\left\langle\gamma(t), \overline{H f_{i}}\right\rangle_{L^{2}}=\int_{t-W}^{t} \gamma^{T}(\tau) \overline{H f_{i}}(\tau) \mathrm{d} \tau
$$

Similarly for sensor faults, we have $\left\langle\gamma(t), \overline{g_{j}}\right\rangle_{L^{2}}$ on an interval $[t-W, t]$. In the presence of a fault, the result of the $L^{2}$-inner product will reveal the fault signature that $\gamma(t)$ most closely aligns with, and thus, enable fault identification.

Consider a component fault causing the inductance of $L_{6}$ to reduce by 50 percent. From (4), we have that the faulted dynamics of $x(t)$ contain an additional term $\phi_{i} f_{i}$. Thus, from (8), the error residual vector will contain an additional component in the direction of $\overline{H f_{i}}=[1,0]^{T}$. Indeed, as shown 


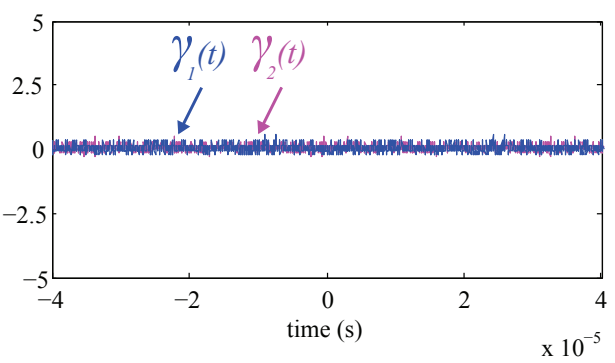

(a) Real-time nominal (fault-free) response.

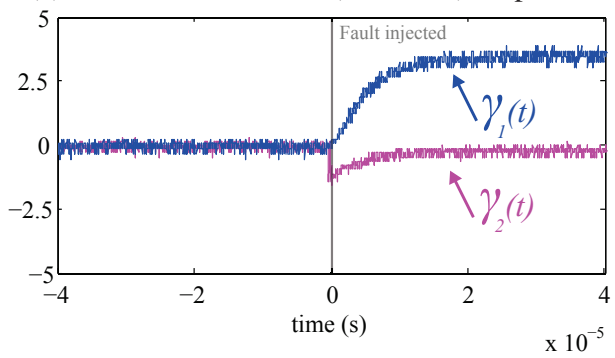

(b) Fault in switch pair forcing $S W_{6, t} \rightarrow$ 'off' and $S W_{6, b} \rightarrow$ 'on'.

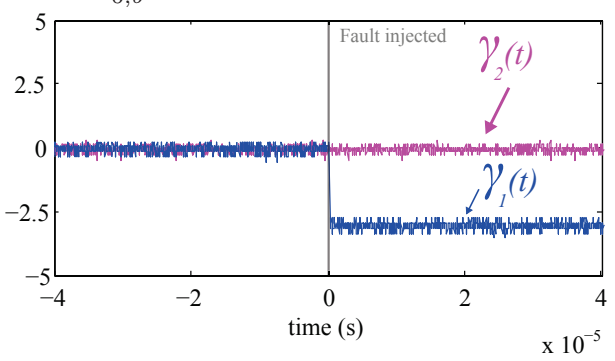

(c) Fault forcing sensor gain of $i_{i n}$ to zero.

Fig. 3: Experimental results of real-time estimator response in nominal and component and sensor fault states.

in Fig. 2b, we see that when the fault is injected at $t=0.02 \mathrm{~s}$, $\gamma_{1}(t)$ becomes nonzero and $\gamma_{2}(t)$ remains essentially zero.

We can follow a similar procedure to identify $\overline{H f_{i}}$ for faults occurring in the output capacitor $C$ and in the switch pair $S W_{n, t / b}$, as shown in Table VI. We see in Figs. 2c and $2 \mathrm{~d}$ that in the faulted steady state, $\gamma(t)$ contains the respective $\overline{H f_{i}}$ components.

Now, consider a sensor fault that forces the sensor gain of $i_{i n}$ to zero. From (6), we see that the faulted dynamics of $y(t)$ contain an additional term $\theta_{j} g_{j}$. Thus, from (8), the error residual vector in steady state will contain an additional component in the direction of $\overline{g_{j}}=[1,0]^{T}$. As shown in Fig. 2e, we see that when the fault is injected at $t=0.02 \mathrm{~s}$, $\gamma_{1}(t)$ becomes nonzero and $\gamma_{2}(t)$ remains zero.

\section{REAL-TIME EXPERIMENTAL IMPLEMENTATION}

In this section, we present an experimental implementation of the model-based estimator for fault diagnosis for the racklevel UPS module. The full specifications for the experimental testbed are presented in Table I.

\section{A. Real-time implementation on FPGA-based control platform}

A single field programmable gate array (FPGA) device performs the converter control and solves the model-based estimator from (7) and (8) in real-time. The FPGA platform is designed for low-latency execution of switched linear state space models of switching power converters (see [13]).

The model-based estimator is solved in discrete time with a fixed 500 ns time step, including input-output latency. Measurements from the converter (i.e. $v_{\text {batt }}(t)$ and $i_{\text {in }}(t)$ ) are sampled with a 16-bit ADC at $1 \mathrm{MHz}$ for control and estimation purposes. The FPGA generates a $50 \mathrm{kHz}$ PWM signal for converter control (i.e. $k(t)$ ) with a 32-bit internal counter, which is directly passed to estimator computation.

\section{B. Results and discussion}

First, we test the nominal (fault-free) response of the estimator as shown in Fig. 3a. As expected, the error residual vector remains essentially zero in steady state.

Next, we inject a fault in the switch pair $S W_{6, t / b}$ that replicates the switch fault presented in Section II-C, that is, $S W_{6, t} \rightarrow$ 'off' and $S W_{6, b} \rightarrow$ 'on' for all $t>0$. The fault is injected into the converter using external MOSFETs that force conduction through the $S W_{6, b}$ path and prevent conduction through the $S W_{6, t}$ path. As shown in Fig. 3b, when the fault is injected at $t=0$, the error residual vector $\gamma(t)$ becomes non-zero in $500 \mathrm{~ns}$, which enables fault detection. Moreover, $\gamma(t)$ evolves in the direction of the normalized component fault signature $\overline{H f_{i}}$ predicted in Table VI.

Finally, we inject a fault that forces the sensor gain of $i_{\text {in }}$ to zero, as in Section II-C. As shown in Fig. 3c, when the fault is injected at $t=0, \gamma_{1}(t)$ becomes nonzero in $500 \mathrm{~ns}$ and $\gamma_{2}(t)$ remains zero, as predicted by the normalized sensor fault signature $\overline{g_{j}}$ in Table VI.

Generally, the time to fault detection is on the order of magnitude of the estimator solver time step (500 ns). Fault identification for various component and sensor faults requires the $L^{2}$-inner product calculation whose solution reaches steady state in around $1 \mathrm{~ms}$. Moreover, the fault diagnosis framework is flexible in that additional component and sensor faults can be accounted for by including the appropriate normalized fault signature.

\section{Generalizations FOR ARbitrary SWitching POWER CONVERTERS}

In this section, we generalize the methodology presented in Section II as to demonstrate the applicability of the proposed fault diagnosis method to a broad class of switching power converters.

\section{A. System and fault modeling framework}

First, we present a general modeling framework to describe the dynamics of the system under nominal and faulted conditions. The large-signal dynamics of a switching power converter can be represented as a switched linear system, that is, a collection of topological configurations coupled with a switching signal, which specifies the active topological configuration at a given time instance. Let $\mathcal{P}$ indicate the set of feasible configurations of a switching power converter. 
The dynamics of each configuration can be modeled in statespace form by applying Kirchhoff's laws to the resulting linear circuit. The active configuration of the system can be uniquely defined by a switching signal function $\sigma(t)$, which is the map $\sigma:[0, \infty) \rightarrow \mathcal{P}$. We can develop the switching power converter model as follows:

$$
\begin{aligned}
\frac{d x(t)}{d t} & =A_{\sigma(t)} x(t)+B_{\sigma(t)} u(t), \\
y(t) & =H x(t)
\end{aligned}
$$

where $x(t)$ is the state vector, $y(t)$ is the output readout vector, $u(t)$ is the input vector, and $A_{\sigma(t)}, B_{\sigma(t)}$, and $H$ are the statespace dynamics of the active configuration indicated by the switching signal $\sigma(t)$ [14].

Next, we present the model for the dynamics of the system under faulted conditions. The faulted dynamics are modeled as the product of a scalar fault magnitude function and a vector fault signature. This product can be summed with the nominal system dynamics to describe the dynamics of the system in the faulted state. It is important to note that the fault magnitude function and fault signature are not linearly additive terms, and instead can be affine functions of $t, \sigma(t), x(t)$, and $u(t)$.

We define two possible classes of faults: (1) component faults, that is, faults that manifest as changes in $A_{\sigma(t)}$ and $B_{\sigma(t)}$, and (2) sensor faults, that is, faults that manifest as changes in $H$.

1) Component faults: Consider the class of possible component faults, the $i^{\text {th }}$ of which is described by a scalar component fault magnitude function $\phi_{i, \sigma(t)}(t, x(t), u(t))$ and a vector component fault signature $f_{i}$. The dynamics of the switching power converter in the presence of this fault can be described as:

$$
\frac{d x(t)}{d t}=A_{\sigma(t)} x(t)+B_{\sigma(t)} u(t)+\phi_{i, \sigma(t)} f_{i}
$$

where $x(t), y(t), u(t), A_{\sigma(t)}$, and $B_{\sigma(t)}$ are as above.

2) Sensor faults: Sensor faults differ from component faults in that they affect elements of $H$ instead of elements of $A_{\sigma(t)}$ and $B_{\sigma(t)}$. Consider the class of possible sensor faults, the $j^{\text {th }}$ of which is described by a scalar sensor fault magnitude function $\theta_{j, \sigma(t)}(t, x(t), u(t))$ and a vector sensor fault signature $g_{j}$. The dynamics of the switching power converter in the presence of this fault can be described as:

$$
y(t)=H x(t)+\theta_{j, \sigma(t)} g_{j}
$$

where $x(t)$ and $H$ are as above.

\section{B. Estimator design for fault diagnosis}

Here, we will motivate and present the general design of the model-based estimator for fault diagnosis in switching power converters.

1) Properties for fault diagnosis: A fault diagnosis system generally has two essential functions: fault detection and fault identification. ${ }^{1}$ Fault detection is the process of detecting the occurrence of a fault in a system that produces some undesirable or unintended behavior. Fault identification is the process of classifying the precise fault that occurred and identifying the faulty component or sensor [15].

Consider a switched linear estimator with inputs $u(t), y(t)$, and $\sigma(t)$ that outputs an error residual vector $\gamma(t)$. From the system and fault modeling framework previously developed, we can identify the properties that the error residual vector $\gamma(t)$ must satisfy in order to enable adequate fault detection and identification.

In the fault-free state (i.e. nominal model), the residual is zero in steady state. When the $i^{\text {th }}$ component fault occurs, the residual becomes non-zero, which enables fault detection, and evolves according to the term $H \phi_{i, \sigma(t)} f_{i}$, which enables component fault identification. Similarly, when the $j^{\text {th }}$ sensor fault occurs, the residual becomes non-zero, which enables fault detection, and evolves according to the term $\theta_{j, \sigma(t)} g_{j}$, which enables sensor fault identification.

2) Estimator design and dynamics: To achieve the desired fault diagnosis properties, we propose a switched linear estimator of the following form:

$$
\begin{aligned}
\frac{d \hat{x}(t)}{d t} & =A_{\sigma(t)} \hat{x}(t)+B_{\sigma(t)} u(t) \\
\gamma(t) & =y(t)-H \hat{x}(t)
\end{aligned}
$$

where $\hat{x}(t)$ is the estimated state vector, $\gamma(t)$ is the error residual vector, $u(t), y(t)$, and $\sigma(t)$ are obtained via measurement, and $A_{\sigma(t)}, B_{\sigma(t)}$, and $H$ are as in (10) and (11).

The open loop error dynamics are stable, that is, that the trajectories $\hat{x}(t)$ and $x(t)$ cannot diverge. ${ }^{2}$ Moreover, due to lossiness in the converter and the corresponding model, we can show that $x(t)-\hat{x}(t)$ asymptotically converges to zero in steady state [16].

In the nominal operating state, the dynamics of the error residual vector $\gamma(t)$ are as follows:

$$
\begin{aligned}
\frac{d e(t)}{d t} & =A_{\sigma(t)} e(t) \\
\gamma(t) & =H e(t)
\end{aligned}
$$

where $e(t):=x(t)-\hat{x}(t)$. Since $e(t)$ asymptotically converges to zero, $\gamma(t)$ will converge to zero in the fault-free state, as desired.

Component faults manifest as changes in $A_{\sigma(t)}$ and $B_{\sigma(t)}$. Consider the $i^{t h}$ component fault. The dynamics of the error

\footnotetext{
${ }^{1}$ Some definitions of fault diagnosis include a third function: fault analysis, in which the magnitude and cause of the fault is determined. We include some of these properties, particularly the determination of fault magnitude, in our definition of fault identification.

${ }^{2}$ One can construct a natural Lyapunov function $V(\gamma)$ corresponding to the energy in the increment of the switching converter. Results from [11] prove that $\dot{V}(\gamma) \leq 0$ for a lossy switching converter containing linear passive reactive elements, switching elements, and time-varying sources.
} 
residual vector $\gamma(t)$ in the presence of this fault are as follows:

$$
\begin{aligned}
\frac{d e(t)}{d t} & =A_{\sigma(t)} e(t)+\phi_{i, \sigma(t)} f_{i} \\
\gamma(t) & =H e(t)
\end{aligned}
$$

In this case, the non-zero magnitude of the component fault magnitude function $\phi_{i, \sigma(t)}(t, x(t), u(t))$ causes $\gamma(t) \neq 0$, which enables fault detection. Moreover, $\gamma(t)$ will evolve in the direction of $H f_{i}$. Thus, by computing the $L^{2}$-inner product $\left\langle\gamma(t), \overline{H f_{i}}\right\rangle_{L^{2}}$ on an interval $[t-W, t]$, where $\overline{H f_{i}}$ denotes the normalized $H f_{i}$, we can identify the fault signature that $\gamma(t)$ most closely aligns with, and thus, achieve fault identification.

Sensor faults manifest as changes in $H$. Consider the $j^{t h}$ sensor fault. The dynamics of the error residual vector $\gamma(t)$ in the presence of this fault are as follows:

$$
\begin{aligned}
\frac{d e(t)}{d t} & =A_{\sigma(t)} e(t) \\
\gamma(t) & =H e(t)+\theta_{j, \sigma(t)} g_{j}
\end{aligned}
$$

Again, $e(t)$ will asymptotically converge to zero. Thus, $\gamma(t)$ will asymptotically converge to the sensor fault magnitude function and fault signature $\theta_{j, \sigma(t)} g_{j}$, which enables fault detection since $\gamma(t) \neq 0$. Moreover, we can compute the $L^{2}$ inner product $\left\langle\gamma(t), \overline{g_{j}}\right\rangle_{L^{2}}$ on an interval $[t-W, t]$, where $\overline{g_{j}}$ denotes the normalized $g_{j}$, to identify the appropriate sensor fault.

\section{CONCLUSiOnS}

This paper presented a real-time model-based fault diagnosis method for a rack-level UPS dc-dc converter. We presented the analysis and experimental implementation of the modelbased estimator for a set of component and sensor faults. Moreover, we presented a generalization of the fault diagnosis method as to provide a scalable framework to encapsulate the dynamics of arbitrary converter topologies and faults. In this way, the proposed fault diagnosis method enables a flexible solution for improving reliability and fault tolerance in an array of power electronics applications.

\section{REFERENCES}

[1] R. J. Patton, P. M. Frank, and R. N. Clarke, Fault Diagnosis in Dynamic Systems: Theory and Application. Upper Saddle River, NJ: PrenticeHall, Inc., Oct. 1989.

[2] R. J. Patton, P. M. Frank, and R. N. Clark, Issues of Fault Diagnosis for Dynamic Systems. Springer Publishing Company, Incorporated, Oct. 2010.

[3] J. Chen and R. Patton, Robust Model-Based Fault Diagnosis for Dynamic Systems. Springer Publishing Company, Incorporated, Nov. 2012.

[4] R. de Araujo Ribeiro, C. Jacobina, E. Da Silva, and A. Lima, "Faulttolerant voltage-fed PWM inverter ac motor drive systems," Industrial Electronics, IEEE Transactions on, vol. 51, no. 2, pp. 439-446, 2004.

[5] R. de Araujo Ribeiro, C. Jacobina, E. Cabral da Silva, and A. Lima, "Fault detection of open-switch damage in voltage-fed PWM motor drive systems," Power Electronics, IEEE Transactions on, vol. 18, no. 2, pp. 587-593, 2003.

[6] R. Peuget, S. Courtine, and J.-P. Rognon, "Fault detection and isolation on a PWM inverter by knowledge-based model," IEEE Transactions on Industry Applications, vol. 34, no. 6, pp. 1318-1326, 1998.

[7] A. Yazdani, H. Sepahvand, M. Crow, and M. Ferdowsi, "Fault detection and mitigation in multilevel converter STATCOMs," Industrial Electronics, IEEE Transactions on, vol. 58, no. 4, pp. 1307-1315, 2011.

[8] D. Zhang, F. Wang, R. Burgos, J. Kern, S. El-Barbari, and D. Boroyevich, "Internal fault detection and isolation for paralleled voltage source converters," 2009 Twenty-Fourth Annual IEEE Applied Power Electronics Conference and Exposition, pp. 833-839, Feb. 2009.

[9] K. Levin, E. Hope, and A. Dominguez-Garcia, "Observer-based fault diagnosis of power electronics systems," Energy Conversion Congress and Exposition (ECCE), 2010 IEEE, pp. 4434-4440, 2010.

[10] X. Ding, J. Poon, I. Celanovic, and A. D. Dominguez-Garcia, "Fault detection and isolation filters for three-phase ac-dc power electronics systems," IEEE Transactions on Circuits and Systems I: Regular Papers, vol. 60, pp. 1038-1051, Apr. 2013.

[11] S. Sanders and G. C. Verghese, "Lyapunov based control for switching power converters," IEEE Transactions on Power Electronics, vol. 7, no. 1, pp. 17-24, 1992.

[12] J. Alimeling and W. Hammer, "PLECS-piece-wise linear electrical circuit simulation for Simulink," Proceedings of the IEEE 1999 International Conference on Power Electronics and Drive Systems. PEDS'99 (Cat. No.99TH8475), no. July, pp. 355-360 vol.1, 1999.

[13] D. Majstorovic, I. Celanovic, N. D. Teslic, N. Celanovic, and V. A. Katic, "Ultralow-latency hardware-in-the-loop platform for rapid validation of power electronics designs," IEEE Transactions on Industrial Electronics, vol. 58, pp. 4708-4716, Oct. 2011.

[14] J. G. Kassakian, M. Schlecht, and G. C. Verghese, Principles of Power Electronics. Addison-Wesley, 1991.

[15] S. X. Ding, Model-Based Fault Diagnosis Techniques: Design Schemes, Algorithms, and Tools. Berlin: Springer, 2008.

[16] L. Kamas and S. Sanders, "Parameter and state estimation in power electronics circuits," IEEE Transactions on Circuits and Systems, vol. 40, no. 12 , pp. $920-928,1993$. 